\title{
Performance Evaluation of the Seismic Alert System (SAS) in Mexico City: A Seismological and a Social Perspective
}

\section{Gerardo Suárez, ${ }^{1}$ David Novelo, ${ }^{1}$ and Elizabeth Mansilla ${ }^{2,3}$}

\section{INTRODUCTION}

Mexico City's Seismic Alert System-Sistema de Alerta Sísmica, in Spanish (SAS) — was conceived after the disastrous Michoacán earthquake of 19 September 1985, which severely damaged Mexico City. The Michoacán earthquake demonstrated that earthquakes located at distances of approximately 300 to $450 \mathrm{~km}$ from Mexico City were capable of inflicting considerable damage to the city, with the subsequent loss of lives and major losses in the city's infrastructure.

The SAS is based on the simple observation that the epicenters of the large earthquakes along the Mexican subduction zone that historically have caused damage to Mexico City, and which could inflict similar damage in the future, are located at distances of 300 to $450 \mathrm{~km}$. As a result, the destructive seismic waves generated by these great earthquakes along the Pacific coast take approximately 60 to 80 seconds to reach Mexico City. Under normal circumstances, seismic waves would attenuate and would not produce damage in cities and towns located at these relatively far distances. This is the case with many of the cities and towns located in the vicinity of Mexico City, which normally are not damaged by large earthquakes that take place on the subduction zone.

In the case of Mexico City, however, seismic waves arriving from the coast are amplified by a factor of 100 to 500 in the frequency band of 0.2 to $0.7 \mathrm{~Hz}$ (e.g., Ordaz and Singh 1992). This amplification is the result of the interaction of the incoming seismic waves with the soft, water-saturated clay soils, on which a large part of the city is built. These clay soils are remnants of the lake that existed in the Valley of Mexico, which has been gradually drained to accommodate the growing urban sprawl.

Therefore, Mexico City occupies a unique and advantageous geographical situation for the design and operation of an early earthquake warning system. Early-warning seismic

1. Instituto de Geofísica, Universidad Nacional Autónoma, Mexico

2. Centro de Estudios para el Desarrollo Alternativo, San Miguel de Allende, Mexico

3. Posgrado de Ciencias de la Tierra, Instituto de Geofísica, Universidad Nacional Autónoma, Mexico alert systems implemented in other parts of the world, such as Taiwan or Japan, have only a few seconds from the time a seismic alert is issued to the moment when the destructive seismic energy hits nearby population centers (Wu and Teng 2002; Hoshiba et al. 2008). This is due to the proximity of the seismic sources to the population centers and critical facilities for which the seismic early warning is intended. In contrast, Mexico City presents a situation where a time span of at least 40 seconds is available for the population to take public safety measures before the damaging seismic waves arrive.

Since the SAS was created over 17 years ago, there has been no evaluation of it that takes into account both the seismological and the social impact of the system. The purpose of this paper is to present such an evaluation of the performance of the SAS since its inception in 1991 to date. Our goal is not to describe the structural details of the system or the technology used by the SAS. Espinosa-Aranda et al. (1995; 2009, this issue) present a detailed description of the SAS. Our evaluation is based in a review of the three main elements that compose the SAS: 1) the technical characteristics and geographical distribution of the instruments installed along the coast of Guerrero to detect potentially damaging earthquakes; 2 ) the performance of the system since its inception, including a review of the track record of alerts issued; and 3) the public safety program that should be structured and implemented to take advantage of the SAS alerts to maximize its benefits for society and to regulate its use.

As part of the evaluation, we conducted an opinion survey of the current users of the system. A catalog and categorization of the users was also made on the basis of the type of user or institutional responsibility. The survey and the catalog of SAS users were used in the evaluation to measure the social impact and use of the SAS as a tool for civil protection in the city. Although the SAS alert signals are also received in some other cities, we constrain our study to Mexico City, the main intended beneficiary of the SAS.

It should be emphasized that this study reviews the performance of SAS, the early earthquake warning system that is composed of 12 sensors that cover the so-called Guerrero seismic gap (Figure 1). More recently, a second earthquake 


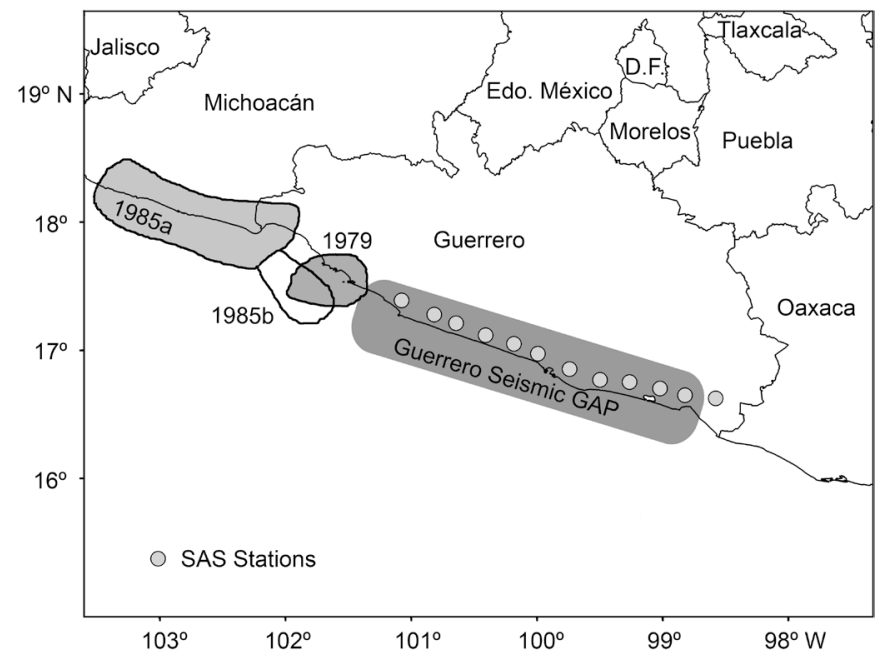

$\Delta$ Figure 1. Location of the 12 strong-motion sensors of the $S A S$ in southern Mexico (filled circles). The shaded areas represent the areal extent of the 1979 Petatlán earthquake, the 1985 Michoacán earthquake mainshock (1985a), its main aftershock (1985b), and the Guerrero seismic gap.

early warning system was installed in the state of Oaxaca, to the southeast of the SAS, which is designed to provide early warning of both coastal and deep in-slab earthquakes that occur inland (Espinosa-Aranda et al. 2009, this issue). This system, called the SASO (Sistema de Alerta Sísmica de Oaxaca), was designed to provide earthquake early warning to Oaxaca City and to the largest population centers in the state. To date, SASO has operated intermittently since its deployment. Both systems, SAS and SASO, now operate independently of one another.

\section{OPERATIONAL BASIS OF THE SAS}

The SAS began to operate in 1991; its first alert was issued for an earthquake of magnitude 4.7 on 16 October 1991. However, the SAS has operated in a formal and uninterrupted manner since 1993 . The 12 strong-motion sensors that monitor earthquake activity along the subduction zone were installed on what has been identified as the Guerrero seismic gap (Figure 1). No large earthquakes, $\mathbf{M}>7.5$, have occurred in this segment of the plate contact since the sequence of large events that struck the coast of Guerrero from 1907 to 1911 (e.g., Nishenko and Singh 1987). Therefore, after the occurrence of the Michoacán earthquake to the northwest, the Guerrero gap was considered the most likely location for the occurrence of a large, damaging earthquake. Although no earthquakes have occurred in the Guerrero gap in the last 100 years, there is ample evidence in the historical record of the presence of large events occurring in this region that are capable of producing damage in Mexico City (García Acosta and Suárez 1996).

The strong motion instruments of the SAS span an area that is approximately $350 \mathrm{~km}$ long in a direction parallel to the coast. The average distance between sensors is $25 \mathrm{~km}$. The spacing guarantees the recording of an earthquake in at least two stations in a time frame of less than about three seconds. The SAS instruments have an algorithm that estimates in an automatic manner the magnitude of the earthquake as it is being generated. The SAS automatically declares an alert when two stations record that a certain acceleration threshold has been exceeded. On the basis of the recorded accelerations, a magnitude is estimated. The alert signals are sent to Mexico City via redundant and independent radio links, in order to ensure robustness and security in the system (Espinosa-Aranda and Rodríguez 2003; Espinosa-Aranda et al. 1995).

Through the end of April 2009, the SAS has emitted a total of 66 alerts based on approximately 2,700 strong-motion records. Of these seismic alerts, 53 have been issued as "preventive alerts" and 13 as "public alerts." A preventive alert is emitted when the magnitude estimated for the earthquake is between 5 and 6. Public alerts, on the other hand, are given when the magnitude estimated is $\mathbf{M}>6$. Earthquakes of magnitudes $\mathbf{M}<5$ should not generate a seismic alert. The SAS does not offer a definition of the magnitude scale used in its estimations.

Under the current scheme, the preventive alerts are sent only to all registered users of the SAS, who have purchased the receiver specifically designed to receive the alerts, with the exception of the local radio and television stations that are users of the system and the metropolitan subway system (Metro). In the case of a public alert, the warning signal is sent to all users. In this latter case, the television and radio stations associated with the SAS interrupt their programming to transmit the alert as a distinct sound signal.

\section{THE SEISMIC ALERT SYSTEM: PERFORMANCE AND STATISTICS}

In order to evaluate the accuracy of the detection algorithms and the validity and usefulness of issuing two different types of seismic alerts, it is important to make a statistical review of the results obtained between the inception of the system and April 2009. Iglesias et al. (2007) also made an evaluation of the algorithm used by the SAS to emit the seismic alerts. The results of our analysis indicate that of the 13 public alerts emitted in the history of the SAS, only three correspond to the SAS's definition of earthquakes with $\mathbf{M}>6$ (Table 1, shaded seismic events). Of the remaining 10 public alerts, six should have been issued as preventive alerts, because they correspond to earthquakes with magnitudes of $5<\mathbf{M}<6$, and the remaining four should not have been issued at all, because the earthquakes in question had in all cases a magnitude smaller than 5. In order to make the comparison between the types of seismic alerts broadcast by the SAS, the reference magnitudes used here are those reported by the Servicio Sismológico Nacional (the Mexican National Seismological Service) and the Global CMT catalog (http://www.globalcmt.org/CMTsearch.html).

On the other hand, since the operational beginning of the SAS, a total of 53 "preventive alerts" have been issued. Of these 53 , only 18 corresponded to the magnitude range used in the definition of preventive alerts (Table 2 , shaded earthquakes). 


\begin{tabular}{|c|c|c|c|c|}
\hline \multicolumn{5}{|c|}{$\begin{array}{c}\text { TABLE } 1 \\
\begin{array}{c}\text { Earthquakes Corresponding to Alerts Issued by the SAS } \\
\text { as Public Alerts }\end{array}\end{array}$} \\
\hline Date & $\begin{array}{l}\text { Origin Time } \\
\text { (GMT) }\end{array}$ & $\mathbf{M}(\mathbf{S A S})^{+}$ & $M_{w}^{*}$ & $\begin{array}{c}\text { Type of Alert } \\
\text { Broadcast }\end{array}$ \\
\hline $06 / 11 / 2007$ & $06: 35: 42$ & 5.6 & & Public \\
\hline $13 / 04 / 2007$ & $05: 42: 22$ & 6.3 & 6 & Public \\
\hline $08 / 10 / 2001$ & 03:39:19 & 5.5 & 5.8 & Public \\
\hline 17/07/1998 & $11: 18: 04$ & 4.6 & & Public \\
\hline 05/07/1998 & 19:55:07 & 4.9 & 5.3 & Public \\
\hline $22 / 12 / 1997$ & 05:22:07 & 4.6 & & Public \\
\hline $13 / 03 / 1996$ & $21: 04: 19$ & 5.1 & 5.1 & Public \\
\hline $16 / 09 / 1995$ & $03: 20: 06$ & 5.0 & & Public \\
\hline $14 / 09 / 1995$ & $14: 04: 30$ & 7.3 & & Public \\
\hline $15 / 05 / 1993$ & $08: 26: 32$ & 4.8 & & Public \\
\hline $15 / 05 / 1993$ & $03: 11: 56$ & 6.0 & & Public \\
\hline $15 / 05 / 1993$ & 03:09:39 & 5.8 & & Public \\
\hline $09 / 11 / 1992$ & 02:13:24 & 4.3 & & Public \\
\hline \multicolumn{5}{|c|}{$\begin{array}{l}\text { + Magnitude reported by the Servicio Sismológico } \\
\text { Nacional, according to the SAS Web page } \\
\text { * Magnitude reported by the Global CMT Catalog } \\
\text { Earthquakes of } \mathbf{M}>6 \text { that were correctly issued as public } \\
\text { alerts are shaded in gray }\end{array}$} \\
\hline
\end{tabular}

Of the remaining events, four should have warranted a public alert as the earthquakes were of $\mathbf{M}>6$, and the remaining 31 earthquakes were in all cases smaller than magnitude 5 and should not have generated an alert.

In summary, the percentage of accurate magnitude broadcasts of either preventive or public alerts is on the order of $32 \%$ (Table 3). As discussed in more detail below, these "errors" in magnitude estimation are not surprising and should not be taken as proof that the SAS does not meet its primary responsibility, i.e., issuing seismic alerts for events that take place on the Guerrero seismic gap and which may damage Mexico City. These results simply indicate that the broadcast of two types of seismic alerts is not supported by the magnitude estimation accuracy of the algorithm used today by the SAS.

Besides these 66 events for which an alert has been issued, since 1992 to date 15 earthquakes have occurred in the vicinity of the Guerrero seismic gap for which the SAS did not generate a seismic alert (Table 4). About half of these events were located on the edges or outside the area covered by the instrumentation of SAS (shaded events in Table 4). The rest are all earthquakes with magnitudes smaller than $\mathbf{M}$ 5.5. The historical and the instrumental records show that subduction earthquakes smaller than M 5.5 do not produce damage or loss of lives in Mexico City.

At first sight, the statistics discussed above appear to be very poor. However, estimating the precise eventual magnitude of an earthquake within the first few seconds of the earthquake generating process is a complex and difficult exercise. Clearly, the magnitude determination of seismic events along

\begin{tabular}{|c|c|c|c|c|}
\hline \multicolumn{5}{|c|}{$\begin{array}{l}\text { TABLE } 2 \\
\text { Earthquakes for Which a Preventive Alert was Issued by } \\
\text { the SAS }\end{array}$} \\
\hline Date & $\begin{array}{l}\text { Origin Time } \\
\text { (GMT) }\end{array}$ & M (SAS)+ & $\mathbf{M}_{\mathrm{w}}{ }^{*}$ & $\begin{array}{c}\text { Type of Alert } \\
\text { Issued }\end{array}$ \\
\hline $27 / 04 / 2009$ & $16: 46: 27$ & 5.7 & 5.6 & Preventive \\
\hline $27 / 03 / 2009$ & 08:48:16 & 5.3 & & Preventive \\
\hline $11 / 11 / 2008$ & 11:02:06 & 4.7 & & Preventive \\
\hline $28 / 04 / 2007$ & $13: 56: 35$ & 5 & 5.1 & Preventive \\
\hline $13 / 04 / 2007$ & 8:43:50 & 5.4 & & Preventive \\
\hline $30 / 03 / 2007$ & $19: 00: 43$ & 4.7 & 5.3 & Preventive \\
\hline $18 / 09 / 2005$ & $11: 25: 53$ & 4.4 & & Preventive \\
\hline $09 / 01 / 2003$ & 02:08:02 & 4.9 & 5.2 & Preventive \\
\hline $27 / 09 / 2002$ & 07:04:58 & 4.9 & 5.1 & Preventive \\
\hline $25 / 09 / 2002$ & $18: 14: 48$ & 5.3 & 5.3 & Preventive \\
\hline $17 / 02 / 2002$ & 04:21:20 & 3.5 & & Preventive \\
\hline $06 / 03 / 2001$ & $21: 57: 56$ & 5.1 & & Preventive \\
\hline $05 / 03 / 2001$ & 10:17:36 & 4.9 & & Preventive \\
\hline $14 / 04 / 2000$ & 01:45:05 & 4.4 & & Preventive \\
\hline $17 / 03 / 2000$ & $00: 50: 58$ & 4.7 & & Preventive \\
\hline $15 / 06 / 1999$ & $20: 42: 07$ & 6.4 & & Preventive \\
\hline $30 / 05 / 1999$ & $09: 58: 42$ & 4.6 & & Preventive \\
\hline 24/04/1999 & 03:08:57 & 4.6 & & Preventive \\
\hline 07/09/1998 & 06:53:17 & 4.1 & & Preventive \\
\hline 09/08/1998 & $16: 18: 08$ & 4.5 & & Preventive \\
\hline 09/05/1998 & 17:03:13 & 4.8 & 5.2 & Preventive \\
\hline $11 / 03 / 1998$ & $14: 13: 12$ & 4.2 & & Preventive \\
\hline 26/08/1997 & $00: 13: 26$ & 4.6 & & Preventive \\
\hline 19/07/1997 & $07: 34: 37$ & 4.9 & & Preventive \\
\hline 14/07/1997 & $01: 26: 19$ & 4.5 & & Preventive \\
\hline $11 / 07 / 1997$ & $22: 23: 34$ & 4.5 & & Preventive \\
\hline $22 / 05 / 1997$ & $07: 50: 55$ & 5.9 & & Preventive \\
\hline 08/05/1997 & $15: 58: 29$ & 4.8 & & Preventive \\
\hline 23/03/1997 & $20: 23: 16$ & 4.7 & & Preventive \\
\hline $21 / 03 / 1997$ & 03:49:16 & 4.6 & & Preventive \\
\hline $11 / 01 / 1997$ & $20: 28: 27$ & 6.5 & & Preventive \\
\hline 27/10/1996 & 09:15:38 & 4.4 & & Preventive \\
\hline $19 / 07 / 1996$ & 09:00:55 & 4.8 & & Preventive \\
\hline 15/07/1996 & $21: 23: 38$ & 6 & 6.6 & Preventive \\
\hline $16 / 09 / 1995$ & $16: 08: 57$ & 4.1 & & Preventive \\
\hline 14/09/1995 & $14: 09: 27$ & 5 & & Preventive \\
\hline $31 / 05 / 1995$ & 12:49:27 & 4.6 & & Preventive \\
\hline 14/04/1995 & 06:01:08 & 4.8 & & Preventive \\
\hline $10 / 12 / 1994$ & $16: 46: 46$ & 6.3 & & Preventive \\
\hline $29 / 10 / 1994$ & $16: 44: 04$ & 5.1 & & Preventive \\
\hline $22 / 05 / 1994$ & 01:41:46 & 5.6 & & Preventive \\
\hline $10 / 09 / 1993$ & $10: 50: 23$ & 4.8 & & Preventive \\
\hline 29/07/1993 & 20:17:01 & 5 & & Preventive \\
\hline $31 / 03 / 1993$ & $10: 18: 15$ & 5.3 & 5.5 & Preventive \\
\hline 4/11/1992 & $04: 33: 37$ & 3.7 & & Preventive \\
\hline
\end{tabular}




\begin{tabular}{|c|c|c|c|c|}
\hline \multicolumn{5}{|c|}{$\begin{array}{c}\text { TABLE } 2 \text { (continued) } \\
\text { Earthquakes for Which a Preventive Alert was Issued by } \\
\text { the SAS }\end{array}$} \\
\hline Date & $\begin{array}{l}\text { Origin Time } \\
\text { (GMT) }\end{array}$ & M (SAS) ${ }^{+}$ & $\mathbf{M}_{\mathrm{w}}{ }^{*}$ & $\begin{array}{l}\text { Type of Alert } \\
\text { Issued }\end{array}$ \\
\hline $30 / 10 / 1992$ & $08: 24: 29$ & 4.4 & & Preventive \\
\hline $16 / 10 / 1992$ & $17: 28: 17$ & 4.3 & & Preventive \\
\hline 02/08/1992 & $12: 54: 42$ & 4.3 & & Preventive \\
\hline 07/06/1992 & $17: 41: 10$ & 4.7 & 5.3 & Preventive \\
\hline 07/06/1992 & 09:01:43 & 4.6 & & Preventive \\
\hline $15 / 05 / 1992$ & 08:35:09 & 4.2 & & Preventive \\
\hline 26/04/1992 & $20: 53: 36$ & 4.3 & & Preventive \\
\hline $16 / 10 / 1991$ & $18: 46: 38$ & 4.2 & & Preventive \\
\hline \multicolumn{5}{|c|}{$\begin{array}{l}{ }^{+} \text {Magnitude reported by the Servicio Sismológico Naciona } \\
\text { * Magnitude reported by the Global CMT Catalog } \\
\text { Earthquakes of }<5 \mathbf{M}<6 \text { that were correctly issued as } \\
\text { preventive alerts are shaded in gray }\end{array}$} \\
\hline
\end{tabular}

\begin{tabular}{|c|c|c|c|}
\hline \multicolumn{4}{|c|}{$\begin{array}{c}\text { TABLE } 3 \\
\text { Statistics of Seismic Alerts Issued by the SAS }\end{array}$} \\
\hline $\begin{array}{l}\text { Type of Alert } \\
\text { Issued }\end{array}$ & $\begin{array}{l}\text { Total Number } \\
\text { of Alerts } \\
\text { Issued }\end{array}$ & $\begin{array}{l}\text { Number } \\
\text { of Correct } \\
\text { Alerts }\end{array}$ & $\begin{array}{c}\text { Percentage } \\
\text { of } \\
\text { Successful } \\
\text { Estimates }\end{array}$ \\
\hline $\begin{array}{l}\text { Preventive } \\
\text { Alerts }\end{array}$ & 53 & 18 & $34 \%$ \\
\hline Public Alerts & 13 & 3 & $24 \%$ \\
\hline Total & 66 & 21 & $32 \%$ \\
\hline
\end{tabular}

the Mexican subduction zone is not the responsibility of SAS. What the statistics show, however, is that given the lack of accuracy in magnitude determination, the differentiation made by SAS in issuing public and preventive alerts is not statistically supported by the results of the analysis and is completely unjustified. Such differentiation between public and preventive alerts is artificial and ineffectual and should be avoided in the future. SAS simply cannot estimate magnitude accurately enough to live by its own definitions of seismic alerts. Instead, a single alert for earthquakes should be issued, raising the threshold to avoid the small events that are felt with low intensity in Mexico City.

The statistics on the performance of the SAS during the past 17 years indicate that although the algorithm used is a poor magnitude estimator, the SAS is capable of broadcasting alerts of potentially dangerous earthquakes to Mexico City occurring along the subduction zone of the coast of Guerrero. During its operational life, the SAS has been capable of correctly identifying all earthquakes with magnitudes greater than 6 and broadcasting an alert. The only exception to this is the earthquake of 18 April 2002 (M 6.7), for which an alert was not issued (Table 3). This earthquake occurred on the northeastern edge of the SAS system of sensors.

\begin{tabular}{|c|c|c|c|c|}
\hline \multicolumn{5}{|c|}{\begin{tabular}{|c} 
TABLE 4 \\
$\begin{array}{c}\text { Earthquakes } M \\
\text { which Did Not Generate a Seismic Alert by the SAS }\end{array}$
\end{tabular}} \\
\hline Date & $\begin{array}{l}\text { Origin Time } \\
\text { GMT }\end{array}$ & $\begin{array}{l}\text { Latitude } \\
\text { (N) }\end{array}$ & $\begin{array}{l}\text { Longitude } \\
\text { (W) }\end{array}$ & $\mathrm{M}_{\mathrm{w}}{ }^{*}$ \\
\hline $12 / 02 / 1992$ & $11: 57: 00$ & 17.78 & 101.14 & 5.2 \\
\hline 31/03/1992 & $20: 56: 41$ & 16.99 & 100.65 & 5.4 \\
\hline 24/10/1993 & $07: 52: 23$ & 16.77 & 98.61 & 6.6 \\
\hline 13/11/1993 & 00:16:59 & 16.28 & 98.61 & 5.7 \\
\hline 18/07/1996 & 08:16:47 & 17.35 & 101.02 & 5.4 \\
\hline 16/12/1997 & $11: 48: 38$ & 16.43 & 98.73 & 5.9 \\
\hline 16/05/1998 & $17: 41: 59$ & 17.27 & 101.34 & 5.2 \\
\hline 11/07/1998 & $05: 21: 15$ & 17.28 & 101.17 & 5.4 \\
\hline 12/07/1998 & $08: 11: 29$ & 16.78 & 99.91 & 5.5 \\
\hline $29 / 10 / 2001$ & $05: 23: 18$ & 17.49 & 99.44 & 5.0 \\
\hline $18 / 04 / 2002$ & 05:03:16 & 16.79 & 101.22 & 6.7 \\
\hline $18 / 04 / 2002$ & 11:00:41 & 16.76 & 100.9 & 5.1 \\
\hline $30 / 08 / 2002$ & 02:11:47 & 16.83 & 100.53 & 5.2 \\
\hline 01/01/2004 & $23: 31: 53$ & 17.45 & 101.40 & 6.0 \\
\hline $19 / 04 / 2007$ & 10:02:14 & 17.26 & 101.03 & 5.3 \\
\hline \multicolumn{5}{|c|}{ * Magnitude determined by the Global CMT Catalog } \\
\hline
\end{tabular}

In summary, the SAS has been capable of identifying all the large events occurring in the area where its instruments are deployed. It may be advisable to modify the algorithm used to make it more robust in eliminating smaller events, establish a magnitude threshold of $\mathbf{M}$ between 5.5 and 6, and broadcast a single type of alert.

\section{INSTRUMENTATION AND GEOGRAPHICAL COVERAGE OF THE SAS}

As discussed before, the SAS was originally designed to provide an early warning for large earthquakes that would presumably take place on the Guerrero seismic gap. Deployment of instrumentation was constrained to the area identified as the gap. Since its inception, the instrumentation has gone through various stages of modernization. The strong-motion instruments and the communication links to Mexico City were designed and built by the Centro de Investigación y Registro Sísmico (CIRES), the institution responsible for operating and maintaining SAS. The equipment used has proven to be very robust and very reliable. No seismic alerts have been missed due to down times or equipment failures.

The digitizers were originally of 12-bit resolution. They have been gradually upgraded to 18 -bit resolution in many stations, providing a greater dynamic range. As we discuss later, the vast majority of the registered users of SAS concede that the system is very reliable. Unfortunately, the accelerometers used by the SAS have a very narrow frequency band. The sensors are of the type commonly used for industrial applications and have also a limited dynamic range. 
It may be advisable in the future modernization plans that the SAS is currently pursuing (Espinosa-Aranda 1995; 2009, this issue) to replace the current accelerometers with modern strong-motion sensors, which offer a much wider frequency band and a larger dynamic range. The inaccuracy observed in the estimation of magnitude may be partly due to the algorithm used and partly to the limited fidelity of the instruments deployed by SAS. Apparently, some records are clipped early on during the earthquake generating process due to the instrumentation used. This assessment is difficult to make in a systematic manner, as the strong motion records generated by SAS are not openly available. The use of high-fidelity accelerometers in the future would also provide an invaluable database for scientists and engineers studying the seismicity of the Mexican subduction zone. Current instrumentation makes the records of little use outside SAS.

In terms of its geographical distribution, the current deployment of sensors covers the area of the subduction zone identified as the Guerrero seismic gap, immediately to the southeast of the rupture of the 1985 Michoacán earthquake (Figure 1). At the time when the SAS was designed, this segment of the subduction zone was considered to represent the fault zone with the highest potential to generate a large and damaging earthquake. Twenty-five years after the 1985 event, this statement is less evident, and today it may be advisable to increase the coverage of the SAS farther to the northwest, toward the rupture of the $1985 \mathrm{event}$, and to the southeast into northwestern Oaxaca (Figure 1). Historical data indicate that earthquakes producing high intensities in Mexico City occur in the segment of the subduction zone from the region where the 1985 event was located to the border between the states of Guerrero and Oaxaca, on the southern edge of where the current SAS sensors are located (García Acosta and Suárez 1997). Subduction earthquakes farther from this segment of the subduction zone have not produced intensities greater than MMI $=5$ in Mexico City (e.g., Figueroa 1987). Hence, subduction events outside of this area are not important from the point of view of an earthquake early warning seismic system targeted for Mexico City.

For an earthquake early warning system to be effective, the time available between the broadcasting of the warning and the arrival of the first destructive seismic waves should be as long as possible. As discussed before, Mexico City has a unique and very advantageous situation in this respect. If the SAS were to broadcast a single type of alert, it should also reconsider the algorithms used. Several researchers have recently proposed various algorithms for this purpose (Allen and Kanamori 2003; Espinosa-Aranda et al. 1989; Nakamura 1988; Wu et al. 1998; Wu and Kanamori 2005).

The broadcasting of a single alert for potentially damaging earthquakes and the avoidance of issuing alerts for small earthquakes that are hardly felt by the population may be considered as one of the future goals of the SAS. Recently, Iglesias et al. (2007) proposed the use of an algorithm that relates the meansquare acceleration in SAS recording stations to the expected acceleration of a reference strong-motion sensor in the out- skirts of Mexico City, outside the area of soft lakebed deposits. The results presented by these authors show a high degree of reliability in identifying potentially destructive earthquakes. Furthermore, the algorithm of Iglesias et al. (2007) avoids the pitfalls and challenges of estimating magnitudes in the near field. The successful operation and performance of the SAS to date could be improved with better, high-fidelity instrumentation and a more effective detection algorithm that weans out small events that are of no consequence to the mission of an earthquake early warning system.

\section{EVALUATION ON THE SOCIAL IMPACT OF SAS AND THE ASSOCIATED PUBLIC SAFETY MEASURES}

The most notorious deficiency of the SAS is not in its technical operation and performance. The most evident and important shortfall is the very limited number of registered users and the lack of a public safety program that promotes the distribution and responsible use of the seismic alert system for civil protection purposes. After almost 18 years of operation, the SAS has only 230 registered users; 25 are radio and television broadcasting stations, 76 are schools of all levels, 12 are emergency and civil protection agencies, four are offices of the subway system, 79 are government offices, 33 are private institutions, and only one is a residential building. Considering the size of Mexico City these figures are disappointingly low (Figure 2).

Among the major limitations in increasing the number of users of the SAS are probably the high cost of the receivers used and the maintenance fees involved. Recently, the SAS has proposed the use of low cost radios similar to those used to receive weather information and warnings, which represent a low initial investment, are easy to install, and require essentially no maintenance.

In particular, the fact that only 76 schools out of a population of approximately 5,500 schools in Mexico City are using the SAS is totally inadequate. Schools should be the primary users of an earthquake early warning system. They are wellstructured organizations with a stable population; under current law, all schools should have drills in case of emergencies. Schools represent a massive concentration of people and the 40 to 50 seconds available to evacuate them in an orderly fashion could represent a crucial element in saving lives in the case of a destructive earthquake. Recent earthquakes in China and Italy provide the best example of the value of giving schools the best tools and information to protect students in case of emergencies.

The same statement could be made about organizations responsible for dealing with emergencies and civil protection. As an example, Mexico City has 16 counties, and each one of them has a civil protection office. To this number, one may add the number of police stations and fire departments, which could benefit from a seismic alert and the design of adequate operational procedures to take in case of an emergency. The fact that only 12 offices responsible for civil protection take advantage of this early warning system is unacceptable. 


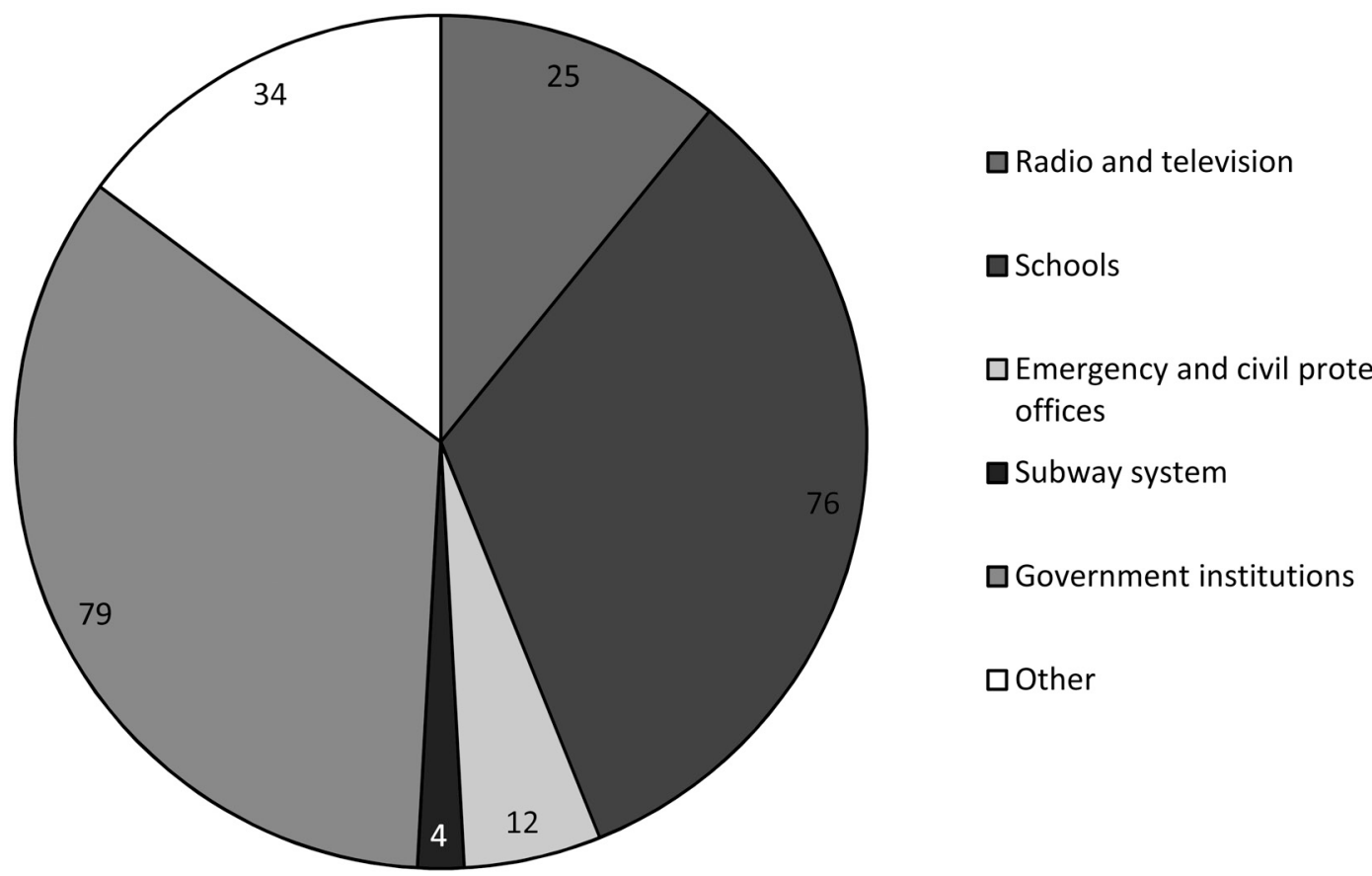

$\Delta$ Figure 2. Number and type of institutions that are registered users of the $S A S$ are shown clockwise: radio and television stations (25), schools (76), emergency and civil protection offices (12), subway system (4), government institutions (79), and private and other users (34).

Unfortunately, there has not been a sustained strategy to increase the number of users or to identify in a structured and organized plan the potential beneficiaries of $S A S$. The system has grown without the benefit of a well-planned program to identify and prioritize users who could make use of this important tool for public safety. The $S A S$ urgently needs a long-term plan that promotes the use of the seismic alert among strategic users and critical facilities in the city. The $S A S$, as a whole, has also lacked the design and testing of operational procedures for users of the system to follow in the case of an alert. Without this social policy, the use and benefits of the $S A S$ will continue to be limited to a small number of users.

In this context, it has to be recognized that 25 radio and television operators automatically interrupt their programs to broadcast the public $S A S$ alerts to the general population that happens to be tuned in to those broadcasters. However, this effort to disseminate the $S A S$ alerts cannot substitute for a structured civil protection program that uses the earthquake early warning signals as a central strategy to save lives. Among experts in civil protection, there has been a debate, ongoing since the early days of the $S A S$, whether broadcasting the signal over a limited number of public and private radio and television stations is an adequate means of communicating an alert to the public and taking full advantage of the $S A S$ (e.g., Fundación Javier Barros Sierra 1992). In our opinion, warning systems of this type should have two populations. The first one is composed of critical facilities such as hospitals, schools, and crucial government offices that adequately understand the limitations and advantages of an earthquake early warning system and are fully trained to take advantage of it. The second is composed of individuals and organizations that decide on their own to receive the early warning signal and decide the best way to make use of it to mitigate the risk.

\section{SURVEY OF THE USERS OF THE SAS}

To obtain the views of the 230 registered users of the $S A S$, we designed a questionnaire and distributed it to all registered users. The questionnaire included questions on the use, efficiency, and quality of the system; the knowledge of the users on the use of a seismic alert; the difference between public and preventive alerts; the reliability of the system; and the existence of manuals and protocols for a particular user to react in case of emergencies, among others (see Appendix). The questionnaire was sent to all users via fax or e-mail, and follow up phone calls were made to prompt users to respond. Out of the 230 users, 131 responded to the survey. To complement the survey a series of private interviews were arranged with institutions representing different families of $S A S$ users.

It is interesting to observe the percentage of answers obtained according to the type of users. If the percentage of users of a certain category answering the questionnaire may be considered as a measure of their interest and commitment, the figures are very revealing (Table 5). The highest percentage of responses came from public elementary schools, private enterprises, and private educational establishments. Somewhat surprisingly, emergency and civil protection agencies, government offices, and television and radio broadcasting stations show a response rate of approximately 50 to $60 \%$.

Ninety-one percent of the users that responded to the questionnaire consider the $S A S$ a useful tool for their institution as a civil protection measure and maintain a positive view 


\begin{tabular}{|lccc|}
\hline \multicolumn{4}{c}{$\begin{array}{c}\text { TABLE 5 } \\
\text { Percentage of Responses Received to the Questionnaire }\end{array}$} \\
\hline Type of Institution & Number of Users & Number of Responses & Percentage \\
\hline Public elementary schools & 27 & 22 & $81 \%$ \\
Private enterprises & 19 & 15 & $79 \%$ \\
Private educational institutions & 49 & 33 & $67 \%$ \\
Emergency and civil protection agencies & 12 & 7 & $58 \%$ \\
Radio and television stations & 25 & 13 & $52 \%$ \\
Metropolitan subway system & 4 & 2 & $50 \%$ \\
Government offices & 79 & 38 & $48 \%$ \\
Residential buildings & 1 & 0 & $0 \%$ \\
\hline
\end{tabular}

of the system as a whole. In general, the users agree that the 50 seconds available between the time the warning is broadcast and the time of the arrival of the strong shaking may represent a key factor in saving lives. Although $91 \%$ of the users claimed to know the $S A S$ well, many of the answer were contradictory. For example, the majority of the users did not know the difference between a public and a preventive alert. Also, many users apparently do not understand that the current system can only provide early warnings for earthquakes occurring on the Guerrero seismic gap and not throughout the Mexican subduction zone. In this context, users lack the information that reporting on other type of events, such as subcrustal earthquakes closer to the city that take place within the subducted Cocos plate, may severely limit the time available to evacuate facilities or take protective measures.

Although users should not be held responsible for understanding the technical and scientific intricacies of the $S A S$, it is important that they are informed of its limitations. In the answers there is the generalized view that the $S A S$ will work for any type of earthquake felt in Mexico City, regardless of the region where it originates. In fact, some users even suggested that the time in which the alert is broadcast should be backed up further to allow more time to evacuate. Users showed in some cases unrealistic expectations of the system based on their lack of information.

In general, the users were very satisfied with the service and maintenance provided by CIRES, the organization responsible for the $S A S$. Users have a very positive view of the service, and almost $90 \%$ of them graded the service provided by CIRES as good or very good. Some users considered the cost of the receivers to be high enough to present an obstacle for public organizations that desire to be part of the system. An important drawback revealed by the survey is that $10 \%$ of the institutions answering the questionnaire lack procedures that regulate their actions in case of an alert. Many of the users claimed to have received no support to develop or implement procedures for evacuation or civil protection in case of emergencies.

According to the survey, users considered the main assets of the system to be the opportunity to be alerted in case of major earthquakes, the possibility of saving lives, the fact that the operation of the system is automatic, and that the system could be used to protect critical facilities in case of an impor- tant seismic event. Users listed as liabilities of the $S A S$ that the receivers were too expensive and technically obsolete; the limited coverage of the seismic alert, which is now constrained to the Guerrero gap; the lack of routinely distributed information on the system; the limited number of users; the lack of information on what to do in case of an alert; and the lack of information and technical support provided by the operators of the $S A S$.

\section{CONCLUSIONS AND RECOMMENDATIONS}

On the basis of the work presented here we come to the following conclusions and present some recommendations with the hope that the $S A S$ may be improved and enhanced in the future.

The $S A S$ has demonstrated in 18 years of operations that it is capable of identifying earthquakes of $M>6$ at an early stage of the fault rupture. The only exception to this is an earthquake that occurred on the edge of the current network on 18 April 2002 (M 6.7). Thus, SAS has demonstrated that it is capable of broadcasting an alert in case of a future large earthquake in the area where its instruments are deployed.

The unique geographical situation of Mexico City allows for an unusually long span of time-about 40 to 50 secondsbetween the broadcast of a seismic alert and the arrival of the first destructive seismic waves. This could make the $S A S$ a very important asset in saving lives and protecting vital installations during a future major earthquake along the Mexican subduction zone south of the city. As such, the $S A S$ should become a key component of the civil protection program of the city in case of a disastrous event.

The algorithm used to estimate magnitude does not have the accuracy to warrant the broadcasting of two different types of alerts-preventive and public_-based on the magnitude of the earthquakes. Based on the results of the 66 alerts issued to date, the approximately $30 \%$ success rate in magnitude estimation shows that separation into two types of alerts is totally artificial and statistically unjustified. Furthermore, there is confusion among the users and general population about the difference between these two types of alerts.

The location of sensors used by the $S A S$ is limited to the so-called Guerrero seismic gap. The network of sensors should 
be extended to include the segment of the subduction zone that has been shown in the past to generate earthquakes damaging to Mexico City. Also, the algorithm used should be adjusted to remove many of the earthquakes of relatively small magnitude $(M<5.5)$ that frequently generate unwarranted seismic alerts.

The number of users is disappointingly low for a metropolis the size of Mexico City. In particular, a very small fraction of the schools, hospitals, emergency relief agencies, and critical lifelines have direct access to the $S A S$ alerts. The low-cost receivers now promoted by the $S A S$ should be used to promote the rational and well-planned use of the earthquake early warning signal as a public safety tool.

After 18 years of continuous operation, the $S A S$ still lacks an integrated plan, in collaboration with local and federal governments, that contemplates not only the technical and scientific issues of an early warning system but includes a structured and planned strategy to identify those institutions, critical facilities, and lifelines that could benefit from SAS early warnings. This plan should not only identify those institutions or facilities that should receive the alert directly, but also prioritize them according to their level of vulnerability (for example, giving priority to those built on the soft, lake deposits of the city) and their need for the alert and capacity to react to it in an organized and structured manner.

Many of the registered users that now receive the $S A S$ alert lack protocols and operational manuals to orderly evacuate their facilities or to take the safety measures necessary in the time span between the alert broadcast and strong shaking to protect lives and property. Current and future users of the system should be helped to develop such procedures according to the type of their institutional organization.

In summary, the $S A S$ has the potential to become a very valuable element in reducing the number of lives that may be lost in Mexico City during a future large earthquake. In order to achieve its full potential, it must strive to broaden its base, convince the authorities to make the $S A S$ one of the cornerstones of the civil protection program of the city, and improve those technical and operational features that have been shown to be less than satisfactory during its operational life.

\section{ACKNOWLEDGMENTS}

This work was done through a contract awarded to Terracon Ingeniería S.A. de C.V. by the Secretaría de Obras y Servicios of the government of Mexico City. We would like to particularly thank Ing. Jorge Arganis for his trust, support, and encouragement during the development of this work. Thanks are also due to J. M. Espinosa-Aranda, director general of CIRES, and to his staff for the information provided during this project. D. Ruiz, C. Sáinz, A. Uribe, and C. Valdes provided valuable information and input during our interviews.

\section{REFERENCES}

Allen, R. M., and H. Kanamori (2003). The potential for earthquake early warning in southern California. Science 300, 786-789.

Espinosa-Aranda, J. M., A. Cuellar, A. Garcia, G. Ibarrola, R. Islas, and S. Maldonado (2009). Evolution of the Mexican Seismic Alert System (SASMEX). Seismological Research Letters 80 (5), 694-706.

Espinosa-Aranda, J. M., A. Jiménez, G. Ibarrola, F. Alcantara, A. Aguilar, M. Henestroza, and S. Maldonado (1995). Mexico City Seismic Alert System. Seismological Research Letters 66, 42-53.

Espinosa-Aranda, J. M., and F. H. Rodríguez (2003). The seismic alert system in Mexico City. In IASPEI International Handbook of Earthquake and Engineering Seismology, ed. W. H. K. Lee, H. Kanamori, P. Jennings, and C. Kisslinger, Part B, 1,253-1,259. New York: Academic Press.

Espinosa-Aranda, J. M., A. Uribe, G. Ibarrola, V. Toledo, and C. Rebollar (1989). Evaluación de un Algoritmo para detectar sismos de subducción. In Memorias VIII Congreso Nacional de Ingeniería Sismica, vol. 1, A199-A211. Acapulco, México: Soc. Mex. Ing. Sismica.

Figueroa, J. A. (1987). Isosistas de grandes temblores ocurridos en la República Mexicana. Instituto de Ingeniería, UNAM.

Fundación Javier Barros Sierra A. C. (1992). El sistema de alerta sísmica en la ciudad de México para sismos generados en la costa de Guerrero, $1^{\text {a }}$. Reunión, internal report.

García Acosta, V., and G. Suárez (1996). Los sismos en la historia de México. Vol. 1. Mexico City: Fondo de Cultura Económica, México D. F., 718 pps.

Hoshiba, M., O. Kamigaichi, M. Saito, S. Tsukuda, and N. Hamada (2008). Earthquake early warning starts nationwide in Japan. Eos, Transactions, American Geophysical Union 89, 73-80.

Iglesias, A., S. K. Singh, M. Ordaz, M. A. Santoyo, and J. Pacheco (2007). The seismic alert system for Mexico City: An evaluation of its performance and a strategy for its improvement. Bulletin of the Seismological Society of America 97, 1,718-1,729.

Nakamura, Y. (1988). On the urgent earthquake detection and alert system (UrErdas). In Proceedings of the 9th World Conference on Earthquake Engineering, Tokyo-Kyoto, Japan, VII, 673-678.

Nishenko, S. P., and S. K. Singh (1987). Conditional probabilities for the recurrence of large and great interplate earthquakes along the Mexican subduction zone. Bulletin of the Seismological Society of America 77, 2,095-2,114.

Ordaz, M., and S. K. Singh (1992). Source spectra and spectral attenuation of seismic waves from Mexican earthquakes, and evidence of amplification in the hill zone of Mexico City. Bulletin of the Seismological Society of America 82 (1), 24-43.

Wu, Y. M, and H. Kanamori (2005). Experiment on an onsite early warning method for the Taiwan early warning system. Bulletin of the Seismological Society of America 95, 347-353.

Wu, Y. M, T. C. Shin, and Y. B. Tsai (1998). Quick and reliable determination of magnitude for seismic early warning. Bulletin of the Seismological Society of America 88, 1,254-1,259.

Wu, Y. M., and T. Teng (2002). A virtual subnetwork approach to earthquake early warning. Bulletin of the Seismological Society of America $92(5), 2,008-2,018$.

\section{Instituto de Geofísica \\ Universidad Nacional Autónoma de México Cd. Universitaria, México D.F. 04510 gerardo@geofisica.unam.mx}

(G.S.) 
APPENDIX

This questionnaire was sent to all Seismic Alert System (SAS) users by e-mail and followed up with individual phone calls to the people responsible for the $S A S$ in each institution.

Institution Name:

Address:

Telephone Number:

Fax Number:

E-mail address:

Person Responsible:

Post:

Date when the SAS receiver was installed at this location

(Day/Month/Year):

1. Do you understand how the SAS operates?

Yes _ No

2. Have you received periodic information about how the SAS operates, the earthquake warnings issued, or on improvements and maintenance of the system?

Yes

No

3. Have you reported failures in the system?

Yes__ No__ Please specify type of failure

4. How would you rate the technical performance of the SAS?

Very good

Good

Fair

Deficient

5. How would you rate the service provided by the Centro de Instrumentación y Registro Sísmico (CIRES), the operators of the SAS?

Very good

Good

Fair

Deficient

6. How much does your institution pay for this service and how often?

Cost $\$$

Frequency of Payments: Monthly

Annual

Other

7. How would you rate the cost paid for the SAS versus the service received by your institution?

Very High

High

Adequate

Low

Briefly specify the reasoning for your answer

8. Since the installation of the SAS at your institution how many earthquake early warnings have you received and of what type? Preventive Public

9. Have you received false alerts?

Yes

On how many occasions?

10. Do you consider useful the issuance of a preventive alert?

Yes

No_ Why?

11. Have personnel in your institution received adequate training on how to proceed after an earthquake early warning is issued? Yes

No

Please specify:

12. Does your institution have regulations and protocols of what to do in case of an earthquake early warning? (For example, evacuation plans, drills, well identified security zones, etc.)

Yes

No

Please specify: 
13. Do you consider it is necessary for your institution to elaborate a plan of action or protocols for this purpose? Yes No Please specify:

14. In case that you have action plans or protocols, were these written in collaboration with CIRES or by your own institution? With support of CIRES

Own efforts Other (specify)

15. During an actual early earthquake warning, have your action protocols operated in an adequate manner? Yes No Why?

16. What type of actions does your institution take when an earthquake early warning signal is issued?

17. Do you consider that the SAS is useful for your institution? Yes_ No N Why?

18. Which do you consider are the main deficiencies of SAS?

19. Which do you consider are the principal advantages of SAS?

20. What recommendations would you make to improve the performance and usefulness of SAS?

21. Do you have any general comments? 